\title{
Animal Experimental Study of the Fully Biodegradable Atrial Septal Defect (ASD) Occluder
}

\author{
Yu-feng Zhu, Xin-miao Huang, Jiang Cao, Jian-qiang Hu, Yuan Bai, Hai-bing Jiang, \\ Zhao-feng Li, Ying Chen, Wei Wang, Yong-wen Qin, and Xian-xian Zhao
}

Department of Cardiology, Changhai Hospital, Second Military Medical University, Shanghai 200433, China

Correspondence should be addressed to Yong-wen Qin, qyongwen@yahoo.com

Received 14 June 2012; Revised 7 August 2012; Accepted 7 August 2012

Academic Editor: George E. Plopper

Copyright (c) 2012 Yu-feng Zhu et al. This is an open access article distributed under the Creative Commons Attribution License, which permits unrestricted use, distribution, and reproduction in any medium, provided the original work is properly cited.

\begin{abstract}
This study was conducted to evaluate the feasibility, safety, biocompatibility, and degradation features of a fully biodegradable occluder for closure of atrial septal defect (ASD) in an acute canine model. The ASD was created in 20 healthy mongrel dogs by the brockenbrough needle, and the fully biodegradable occluders were implanted by self-made delivery system. The success rate and complications were observed. Acute ASD models were successfully created in 18 dogs, and 16 occluders were successfully implanted in the ASD models. Animals were sacrificed at different times after procedure. The cardiac gross anatomy showed that all occluders were stable in the interatrial septum, no vegetation or thrombus formation was observed on the surface of all occluders. They were embedded into endogenous host tissue gradually at 12-week follow-up. Different periods of pathological observations suggested that the occluders degraded gradually over about 24 weeks and essentially became an integral part of the septum. Transcatheter closure of ASD in acute canine model using the fully biodegradable ASD occluder has the potential of a high successful rate of technique, excellent biocompatibility, and fewer complications with adequate, immediate, and short-term results.
\end{abstract}

\section{Introduction}

Atrial septal defect (ASD) is one of the most common congenital heart defect and accounts for about $30 \%$ of the congenital heart disease. With the development of interventions and cardiac catheterization, the interventional treatment has become the preferred option for ASD patients. Currently, nickel-titanium-alloy occluders have been widely used; however, there are many complications involved with it, such as erosion or perforation of the device, delayed endothelialization, thrombus formation, severe valvular damage, hemolytic phenomena, nickel allergy, and even aortic-atrial fistula [1-6]. Therefore, there is a need to find more appropriate materials to reduce such complications. As of now, the percutaneous devices for septal defect treatment are made of nondegradable metallic and synthetic fabric materials. These devices are not ideal due to risks of future complications from device erosions and potential obstructed access for future transseptal procedures [7]. Ideally, occluder material should be completely absorbed by the body as a bridge to achieve the purpose of self-healing defect.
To address this, we selected polydioxanone (PDO), polyL-lactic acid (PLA), polyglycolic acid (PGA), and other biodegradable materials to develop a fully biodegradabl ASD occluder. The study was conducted to evaluate the feasibility, safety, biocompatibility, and degradation features of transcatheter closure of ASD in acute animal (canine) model using the fully biodegradable ASD occluder.

\section{Materials and Methods}

2.1. Occluder Device. The ASD occluder made from biodegradable materials is a self-expandable double-disc device which is similar in construction to the Amplatzer septal occluder (Figures 1(a) and 1(b)). Two-disc-like occluders with $3 \mathrm{~mm}$ waist length were prepared with a $0.298 \mathrm{~mm}$ thickness PDO using monofilament woven technology and thermoforming technology. A flat disc-shaped structure at left side represents the edge slightly to close to the right internal and is approximately $15 \mathrm{~mm}$ in diameter. The right side was disc shaped and approximately $10 \mathrm{~mm}$ in diameter. There was a little sunken groove in the centre and a circular loop laid 


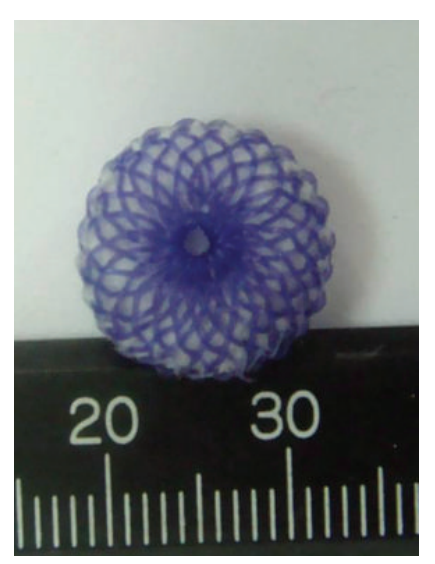

(a)

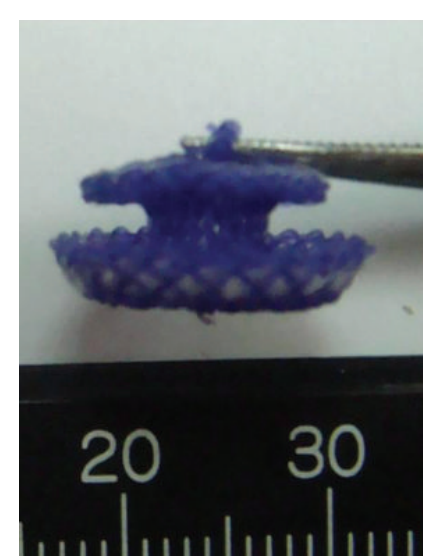

(b)

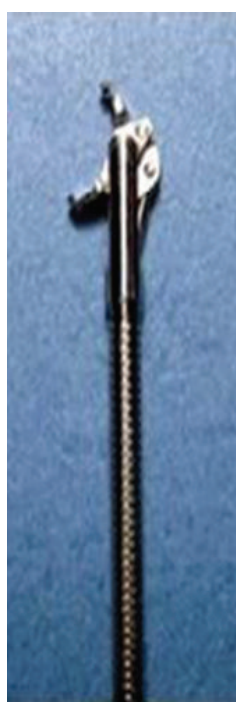

(c)

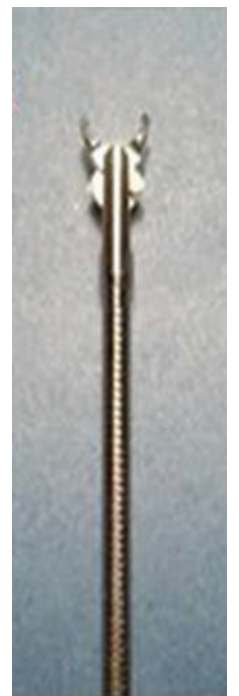

(d)

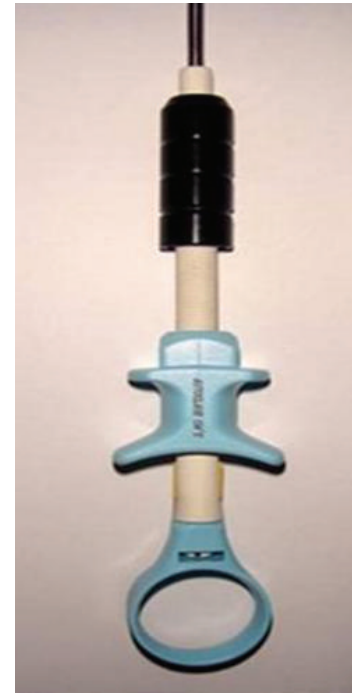

(e)

Figure 1: Biodegradable ASD occluder and delivery system. (a) Left atrial view; (b) lateral view, the end of the circular loop picked up by the tweezer; (c) the jaw of the delivery device was designed to be an unstable structure, and it can be opened on both sides according to the force to prevent hook when the occluder was released; (d) the forward opening of the jaw; (e) propeller type manipulated handle.

hidden inside for transportation. The PDO possesses a good shape memory so that the occluder can be compressed radically and facilitate delivery and release of the transcatheter. Both sides of the discs were filled with woven PLA to block blood flow. Two metal tantalum particles $(0.5 \times 0.5 \times$ $0.5 \mathrm{~mm}^{3}$ ) were fixed at the edge of each side of the discs in symmetrical position, as markers under X-ray.

2.2. Delivery System. The overall structure of the delivery device was similar to a foreign body forceps, with a front hinged jaw and a straight rob to control the opening and closing. The forceps were designed in arc shape to prevent the injury of blood vessels during the opening or closing time. There was a groove on the medial side, and the rear loop of occluder was placed in the groove and so that it would not slip. On the rear side it adopts a spiral design with automatic locking, to manipulate the jaw-opening angle (Figures 1(c) and $1(\mathrm{~d})$ ).

\subsection{Preparation of ASD Model and Transcatheter Closure.} Twenty healthy mongrel dogs weighing 17-22 kg (male and female) were purchased from the experimental animal center of Second Military Medical University. Preoperative auscultation showed no heart murmur. Intramuscular injection of ketamine $(10 \mathrm{mg} / \mathrm{kg})$ and diazepam $10 \mathrm{mg}$ and then an additional intravenous propofol were given according to the actual situation in the surgery. Under X-ray guidance, long guidewire, $8 \mathrm{~F}$ Mullins transseptal sheath, and brockenbrough needle were placed into the superior vena cava by right anterior oblique projection. The puncture needle was withdrawn to select the puncture site in the right anterior oblique in 75 degree position. After puncture, a steel wire was delivered to the left atrial, and heparin was administered. A $14 \mathrm{~F}$ delivery sheath was introduced over the retained wire across the defect. An appropriate occluder was selected; a push rod was used to clamp the circular loop of occluder at the right atrial through the loaded catheter; the fixed screw was tightened in the rear to close the jaw. The occluder was loaded into the catheter, and any air trapped inside was removed. The occluder was connected to the delivery system, and then the occluder was pushed through the delivery sheath into the left atrium under X-ray guidance. The disc was expanded on the left atrial side of occluder in the left atrial cavity (judged by the tantalum markers located in the symmetrical position at the edge of the disc), the sheath was withdrawn, and the device was delivered to make sure that the disc on the left atrial side of occluder was clings to the atrial septal. The delivery device was fixed when resistance was felt during withdrawal then the sheath was withdrawn to open the disc on the right atrial side of occluder. In case of resistance, the closure device was fixed by gentle sliding. Different fluoroscopy positions were taken to ensure complete opening of the disc of occluder, and tranthoracic echocardiography confirmed the location of the occluder in the atrial septal. Both the discs were well opened, and the delivery device was loosened to release the occluder. The catheter was withdrawn, firm pressure was applied to the puncture site, and the closure was completed (Figure 2).

2.4. Postoperative Management. Postoperative antibiotics (penicillin and amikacin) were used to prevent infection for three days. The general state was observed, such as feeding, mental status, living habits, urine, the presence of hemiplegia, and abnormal behavior. The ECG, transthoracic echocardiography, and X-ray were checked periodically. After 2 weeks, 4 weeks, 8 weeks, 12 weeks, and 24 weeks of implantation, 2-3 dogs were sacrificed, and the hearts 


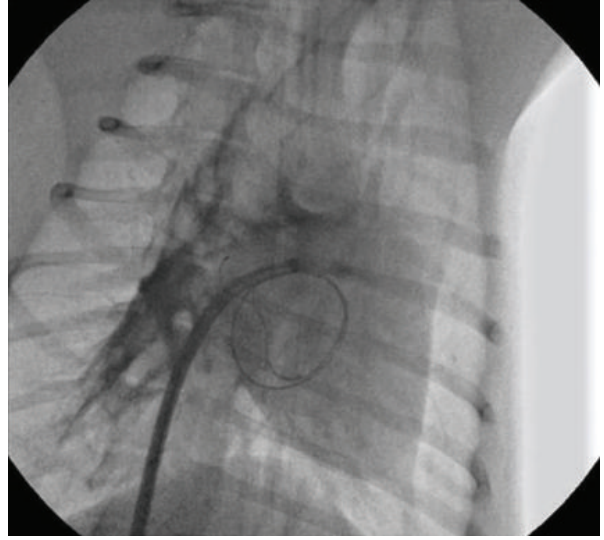

(a)

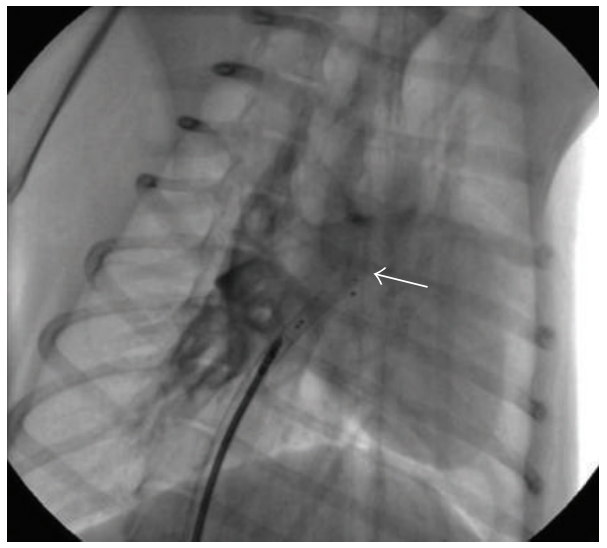

(c)

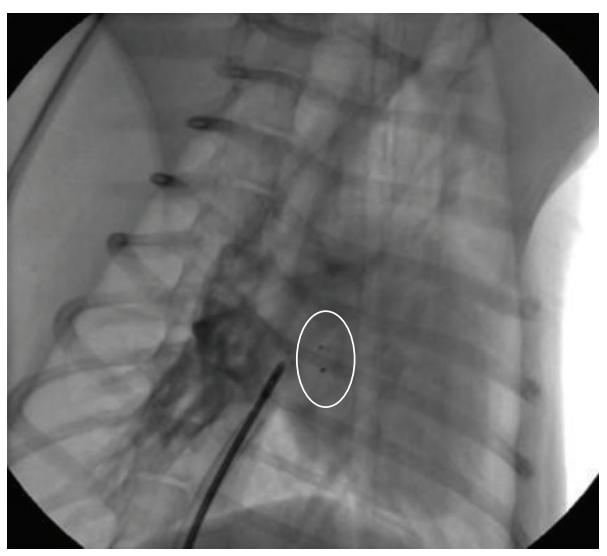

(e)

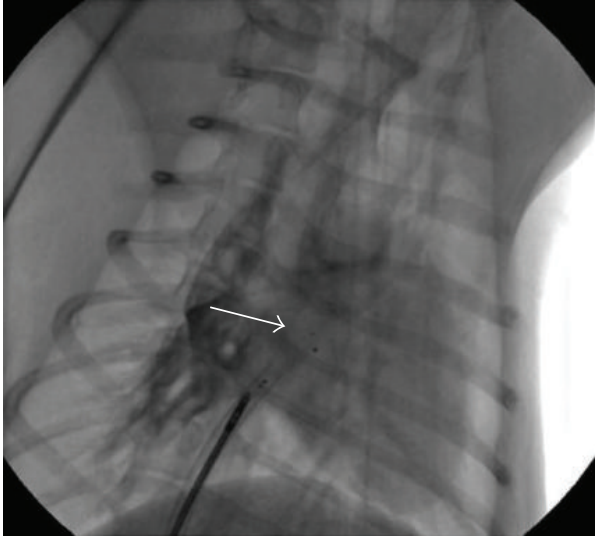

(b)

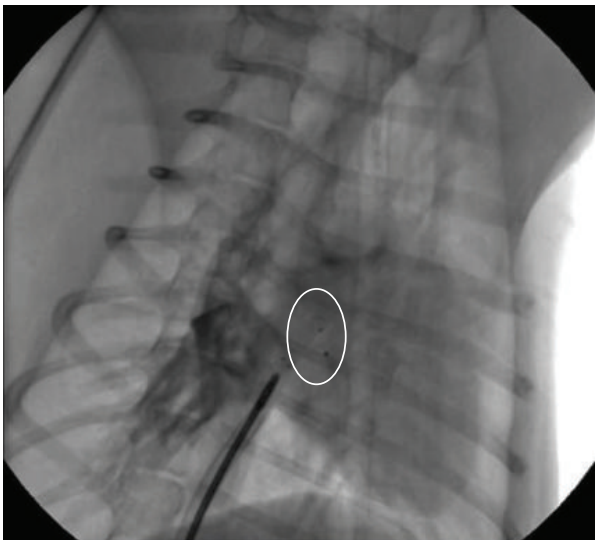

(d)

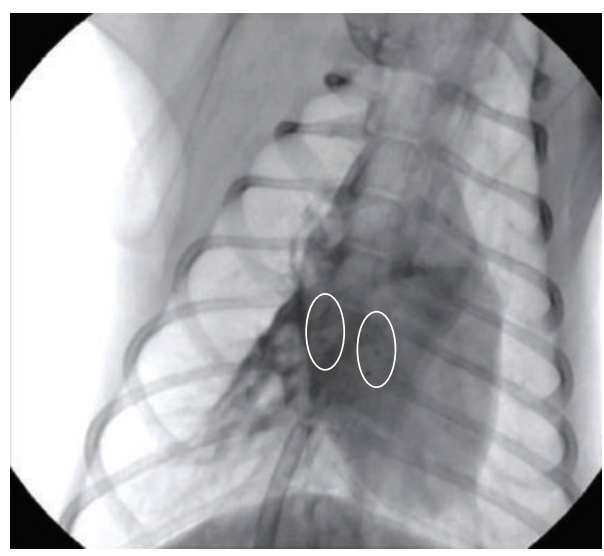

(f)

FIgURE 2: Transcatheter placement of biodegradable ASD occluder (a) retains the guide wire after the model was created; (b) the occluder (indicated by the white arrow) passes through a sheath, and tantalum marker particles can be seen in the front of occluder; (c) unsheathed the left atrial side of occluder (indicated by the white arrow); (d) the release of the left atrial side of occluder, tantalum particles were unsheathed and separated; (e) the release of the right atrial side of occluder, separation of tantalum particles can be seen (see the white oval); (f) after the occluder release, tantalum markers indicate that both of the two discs were well opened (two small white oval).

were removed. Gross anatomical examination was done to observe the occluder and adjacent anatomic structure. The occluder and surrounding tissue were taken for pathological examination to observe the surrounding inflammatory response, tissue hyperplasia, endothelialization, and the degradation of the PLA film and PDO wire of the occluder. Immunohistochemical staining for CD34 was performed on the paraffin-embedded tissue sections. The lung, liver, spleen, and kidney were also removed for pathological examination. After 8 weeks and 12 weeks of implantation, 
endothelialization of occluders was observed with the scanning electron microscopy.

\section{Results}

3.1. Built Models and the Technical Success Rate. The ASD model was successfully created in 18 dogs, and 16 occluders were implanted successfully. The success rate was $88.9 \%$. One case died due to a large quantity of gas in the right ventricular through the delivery sheath during the release of occluder. Implantation was abandoned in another case due to the accidental injury of conduction system which caused the slowdown of heart rate. Sixteen successfully implanted occluders were delivered and released smoothly; the position of occluders was normal; tantalum particles were visible under the X-ray. Intraoperative ECG monitoring showed no other complications except for a transient atrial premature beat. The occluder was clearly visible under ultrasound after release; both the discs were well opened. Color Doppler ultrasound showed that 15 of 16 cases $(93.75 \%)$ had complete closure, and 1 of 16 cases $(6.25 \%)$ had a trace to small shunt immediately after the procedures. There was no mitral regurgitation or tricuspid regurgitation. The total duration of operation including preparation of models and occluder placement was $70 \pm 17.25 \mathrm{~min}(40-94 \mathrm{~min}, n=16)$. The mean X-ray fluoroscopy time was $15 \pm 3.52 \mathrm{~min}(12-$ $22 \min , n=16$ ).

\subsection{Postoperative Followup}

3.2.1. The General State and Examination of Ultrasound, ECG, and $X$-ray. Dogs were in good mental state, eating normally, and exhibited free movements after the procedure. There was no paralysis, abnormal behavior, hematochezia, and gross hematuria. ECG was normal at all-time points, no shift in the tantalum markers was observed under X-ray fluoroscopy. There was no significant mitral regurgitation or tricuspid regurgitation or pulmonary vein regurgitation as reported by ultrasound examination. Echocardiography showed that the occluder was still clearly visible at 8 weeks, but at 24 weeks the occluder remained unidentified (Figure 3 ).

3.3. Macroscopic Examination of Cardiac Anatomy. Animals were sacrificed at different times ( 2 weeks, 4 weeks, 8 weeks, 12 weeks, and 24 weeks). After procedure, the cardiac gross anatomy showed that all occluders were present in the interatrial septum. There were no obvious changes in the shape of the heart, the volume of cardiac chamber and myocardial wall thickness, and there were no valvular lesions. The occluder was implanted more than $2 \mathrm{~mm}$ away from the mitral valve, tricuspid valve, coronary sinus, right superior, and inferior pulmonary vein, wherein no changes in the flow velocity were found. There were no vegetation and thrombus formation on surface of all the occluders. Two weeks after the procedure, the occluder shape was retained, the surface of occluder was covered with a layer of newborn film, PDO wire was clearly visible, and the color was still light blue (Figure 4(a)). Four weeks after the procedure, the surface of

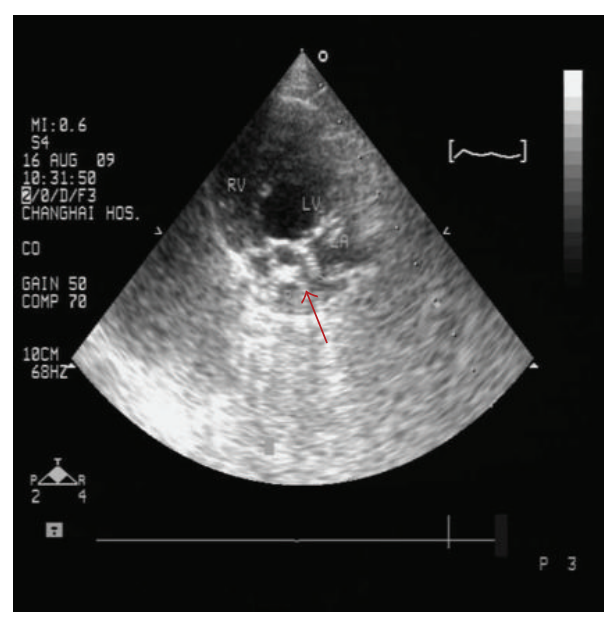

(a)

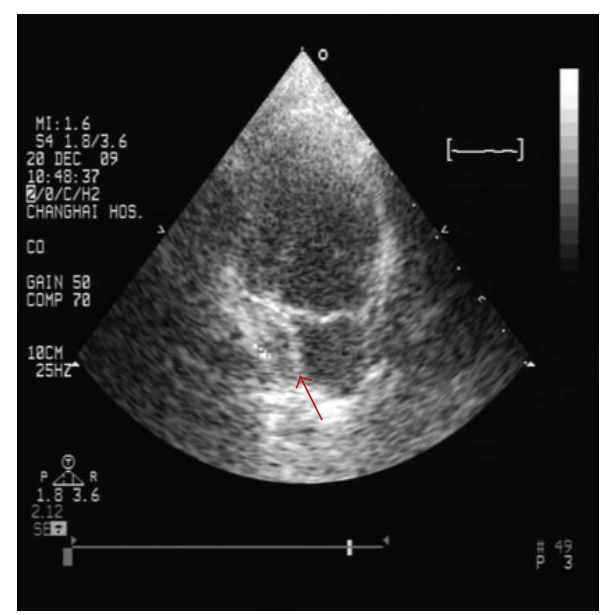

(b)

FIGURE 3: Ultrasound followup. (a) The occluder was still clearly visible in atrial septal at 8 weeks (red arrow); (b) at 24 weeks, the occluder has been unable to identify (the red arrow showing the atrial septal).

occluder was covered with a layer of translucent red film, the shape of the occluder was retained but was partially fused with the endocardial tissue, PDO wire was still clearly visible, and the color was faded (Figure 4(b)). Eight weeks after the procedure, the surface of the occluder was covered with reddish-white film, the PDO wire was still visible but fused with the endocardial tissue, and the occluder shape was still retained (Figure 4(c)). Twelve weeks after the procedure, the surface of the occluder was basically covered with milky white film, mixed with a little red, the PDO wire was faintly visible (Figure 4(d)). Twenty-four weeks after the procedure, the surface of the occluder was covered with a dense porcelainwhite film, the PDO wire was unidentifiable (Figures 4(e) and $4(\mathrm{f})$ ), the occluder was bisected by a symmetrical cut along the long axis of the waist, the occluder became an integral part of the septum, the contour was still visible, but the PDO wire and PLA film were not identifiable. At different time points after the procedure, no abnormalities in the gross 


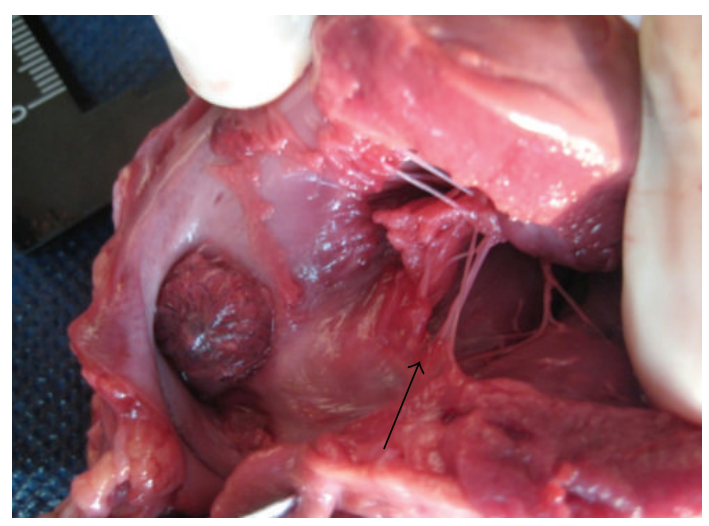

(a)

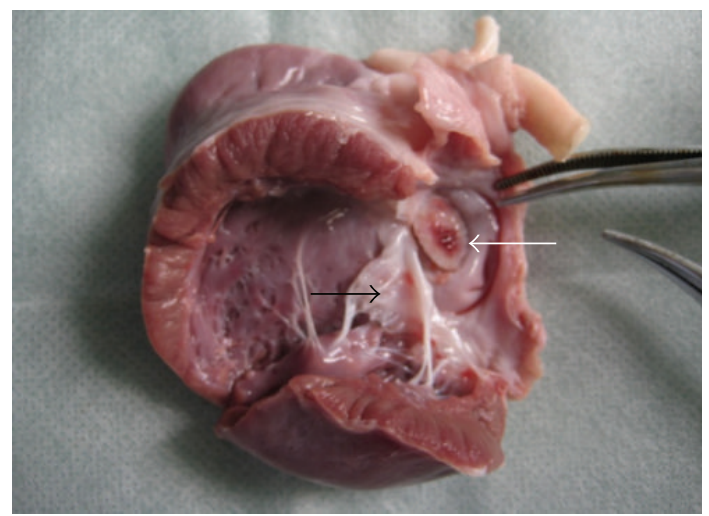

(c)

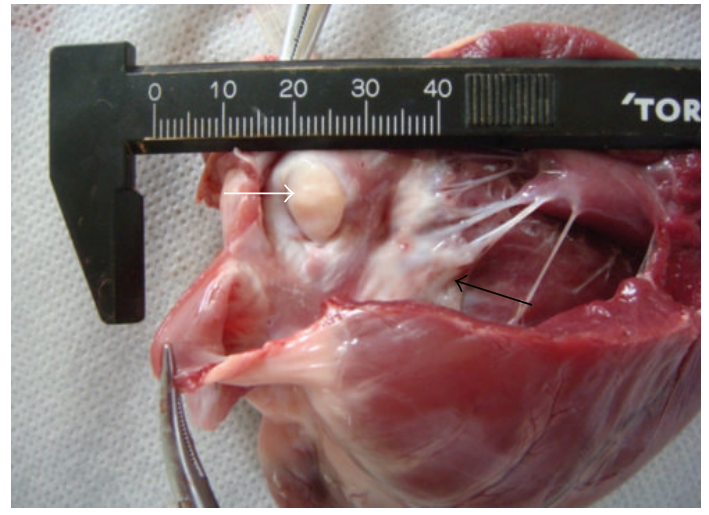

(e)

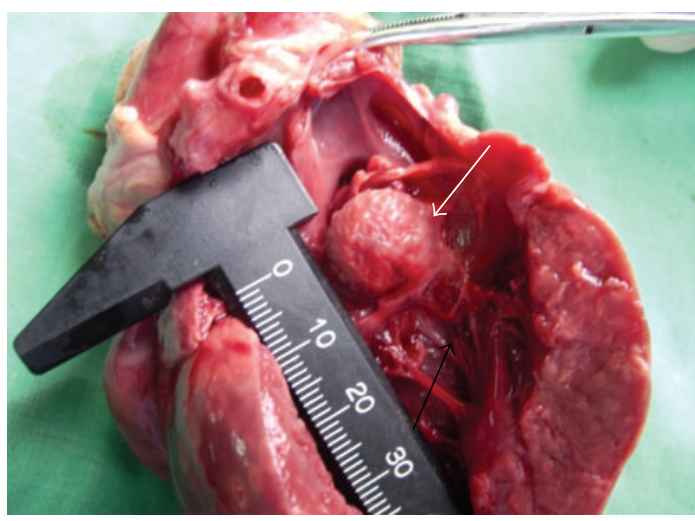

(b)

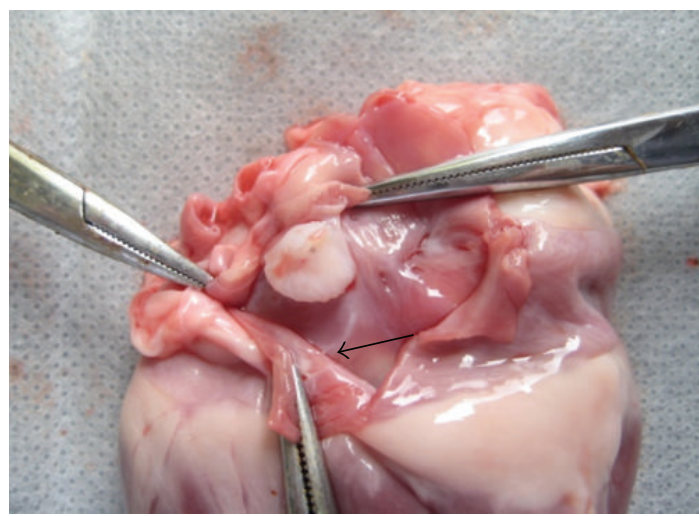

(d)

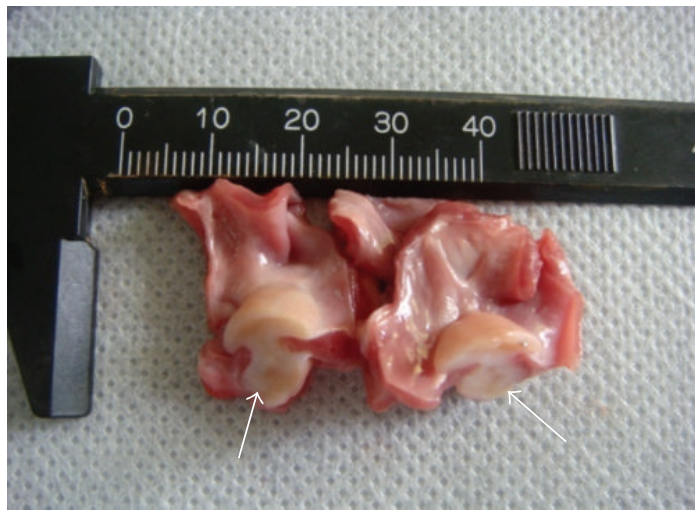

(f)

Figure 4: Gross inspection of the occluder at different time points after procedure (white arrows show the biodegradable occluder, and black arrows for the tricuspid annulus). (a) 2 weeks after procedure, the occluder shape was kept, the surface of occluder was covered with a layer of newborn film, PDO wire was clearly visible; (b) 4 weeks after procedure, the surface of occluder was covered with a certain amount of connective tissue, the occluder shape was kept, and partially fusion with the endocardial, PDO wire was still clearly visible; (c) 8 weeks after procedure, the surface of occluder was covered with reddish-white film, the PDO wire was still visible; (d) 12 weeks after procedure, the surface of the occluder was basically covered with milky-white film, mixed with a little red, the PDO wire was faintly visible; (e) 24 weeks after procedure, the surface of the occluder was covered with a dense porcelain-white film, the PDO wire has been unidentifiable; (f) 24 weeks after procedure, the occluder was bisected by a symmetrical cut, the occluder has become an integral part of the septum, the contour was still visible, but the PDO wire and PLA film have been unable to identify.

specimens of lung, liver, spleen, and kidneys were noted, and no infarction was observed by naked-eye examination.

3.4. Histopathological Examination. Eight weeks after procedure, haematoxylin eosin (HE) staining showed a large number of inflammatory cells around the occlude, PDO and PLA wire retained their shape, obvious fibrous connective tissue hyperplasia was found (Figures 5(a) and 5(b)). Twelve weeks after the procedure, inflammatory response significantly decreased. The PDO wire at the edge of the discs began 
to disintegrate but was still in good shape, there were clear boundaries between PDO wire and surrounding tissues (Figures 5(c) and 5(d)). The surface of occluder was lined with a continuous layer of flattened cells (they were confirmed as endothelial cells by CD34 immunohistochemistry staining) under which fibrous connective tissues, thin-walled neovascular, were seen (Figure 6(c)). Sixteen weeks after procedure, there was no inflammatory response; most of the PDO wire had already disintegrated, and some of it was substituted by hyperplastic fibrous connective tissue; new blood vessels were also found (Figures 5(e) and 5(f)). Twenty-four weeks after the procedure, inflammatory response was completely dissipated, two discs were endothelialized completely with dense fibrous connective tissue at the bottom, thick-walled neovascular, was seen, most of the PDO wire was disintegrated and substituted by hyperplastic fibrous connective tissue, also PLA began to disintegrate (Figures 5(g) and 5(h)). Histopathological observations of lung, liver, spleen, and kidneys were done after procedure at 2 weeks, 4 weeks, 8 weeks, 12 weeks, and 24 weeks; no abnormalities were noted, and no infarction was found.

3.5. Scanning Electron Microscopic Observation. Eight weeks after the procedure, the surface of occluder was lined with a continuous layer of endothelial cells, showed an imbricated surface, but was relatively sparse. There was fibrous connective tissue among the endothelial cells. Twelve weeks after procedure, endothelial cells have become denser, and the surface of occluder showed a wave-like surface (Figures 6(a) and $6(\mathrm{~b}))$.

\section{Discussion}

The Amplatzer septal occluder device is currently the most widely used occluder for transcatheter closure of ASD. However, some potential short comings remain unavoidable. In particular, some amount of cardiac erosion or perforation occurred during late followup and are potentially life threatening, which makes its long-term safety being questionable [4-6]. The mechanism of late-onset cardiac perforation remains unknown. We presumed that the lateonset complication after the occluder deployment may be caused by continuing compression of the adjacent atrial tissue due to the shape memory property of nitinol. Thus, the improvement of production materials would help to reduce complications. In a study by Wu et al., ASD has been termed as the "Chinese Lantern" made of fully biodegradable polymers featured with a unique pull-fold mechanism. The $\mathrm{X}$-ray imaging showed that the devices were in satisfactory position and stable after one month of followup [8].

After implantation, the occluder provides a temporary "bridge" for cardiac self-repair. Once the defect is firmly covered by host connective tissue and endothelial cells, occluder device would lose the value of existence and will not need a permanent retention in vivo. Therefore, an ideal ASD occluder should be absorbed by the body, so that defect could be repaired completely by host organization and should eliminate long-term complications and other unfavorable factors caused by the permanent retention of occluder device.
The BioSTAR septal repair implant (NMT Medical, Boston) is a bioabsorbable device, instead of the polyester fiber membrane of occluder; the device is made of a cellular porcine intestinal type I collagen layer. As compared with nondegradable polyester fiber membrane occluder device, BioSTAR can be absorbed and replaced by host tissue in vivo and has the advantage of reducing thrombogenicity and improving biocompatibility [9-12]. But the device cannot be completely degraded because the BioSTAR possesses the traditional stainless steel framework. A case of late cardiac perforation by metal struts has been reported [13]. The success of development of BioSTAR and clinical trials favoring it mark a huge step forward from metal occluder device to biodegradable occluder device, and bring new ideas and hope to intervention workers. Therefore, the development of biodegradable occluder device will be the development trend in the future.

Synthetic biodegradable polymers have been approved by the US FDA to produce implant materials in humans. These materials have a good biocompatibility, and degradation products do not cause adverse effect on organizations. Our research indicated that biodegradable occluder device made of PLA, PDO, PGA, and tantalum particles has the following advantages. Occluder can achieve a good compressibility and elastic recovery by means of monofilament fiber woven technology. Liu et al. also used nanofibrous matrix of polyD-L-lactide-glycolide (PLGA)/type I collagen blend which was produced via electrospinning to develop biodegradable and biomimetic antishunt membranes for the occluders [14]. Zilberman et al. [15] studied self-expandable tubular stents made of PLA, PDO, and PGA, in vitro degradation and mechanical properties. Research showed that the degradation time of PLA scaffolds were approximately 24 months and can provide effective support for at least 20 weeks; degradation time of PDO was 6 months and can afford good support for 5 weeks; PGA is relatively rapid but can provide good support for only 2 weeks. Occluder should have good compressibility and shape memory properties, in view of that transcatheter delivery, and release is also needed. The monofilament of PDO, which had been woven into biodegradable self-expanding oesophageal stents $[16,17]$ and bronchial stents, [18] combined a high initial strength and modulus together with good ductility and flexibility, and were therefore chosen for use in the occluder to provide a self-expandable property. After the implantation, the time required for full endothelialization and effective support was approximately three months; we therefore adopted PDO to produce the scaffold of cardiac defect occluder and chose PLA film with the degradation time of up to 2 years.

Trancatheter closure of atrial septal defect is conventionally guided by X-ray fluoroscopy, so the occluder should be visible under the X-ray. Biodegradable polymer materials can penetrate the X-ray; however, it is difficult to positioning and release of occluder. To solve the problem, four tantalum particles $\left(0.5 \times 0.5 \times 0.5 \mathrm{~mm}^{3}\right)$ were fixed with PLA absorbable suture at the edge of each side of the discs in symmetric position to achieve the purpose of imaging tracer under DSA. Our experiment confirmed that the location of occluder could be investigated by imaging the tantalum 


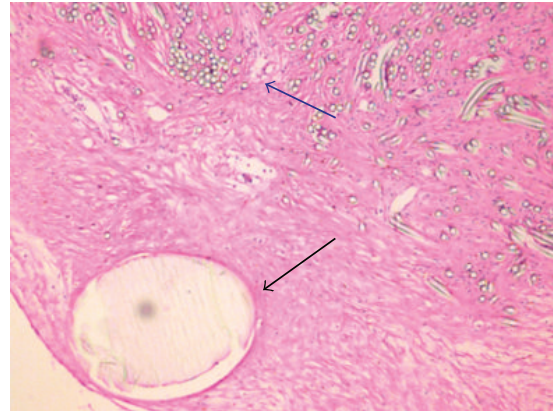

(a)

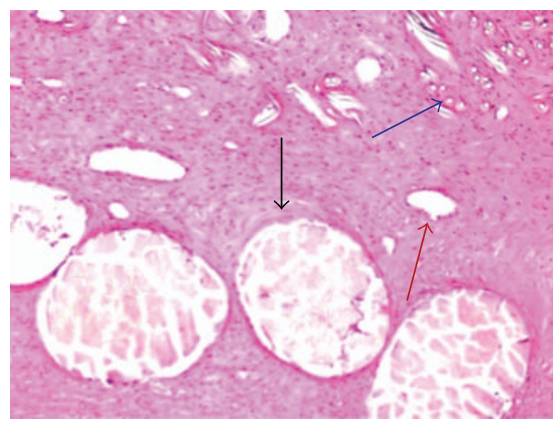

(c)

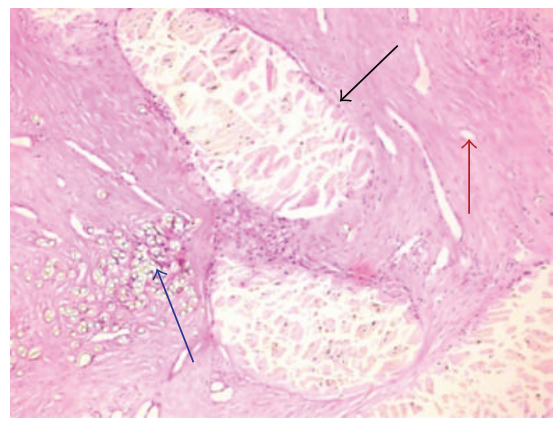

(e)

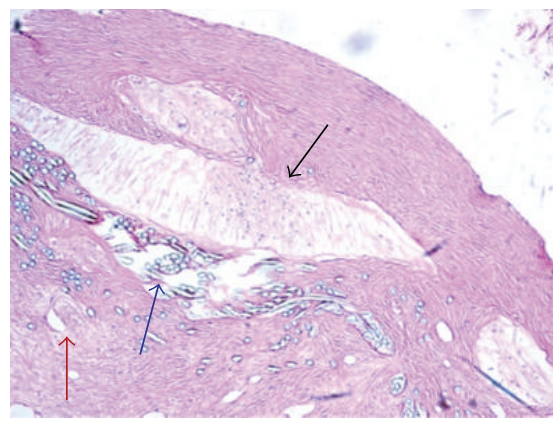

(g)

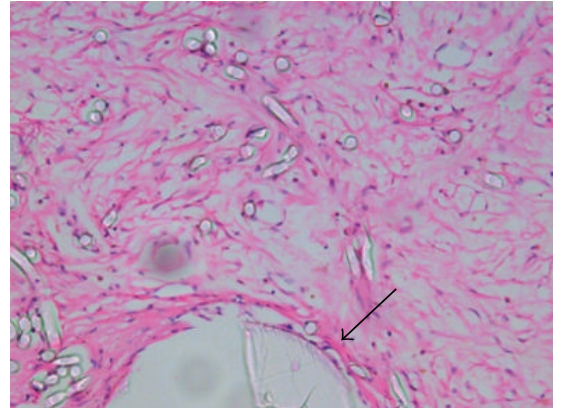

(b)

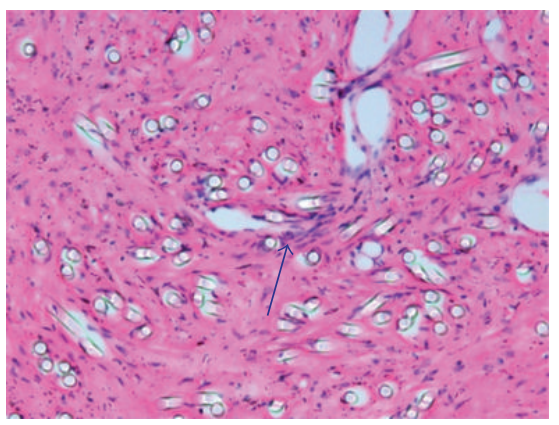

(d)

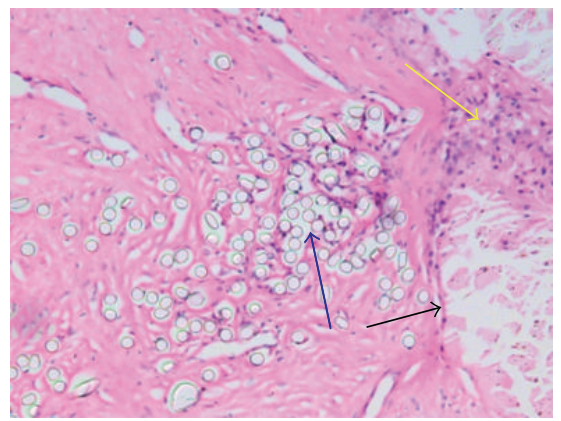

(f)

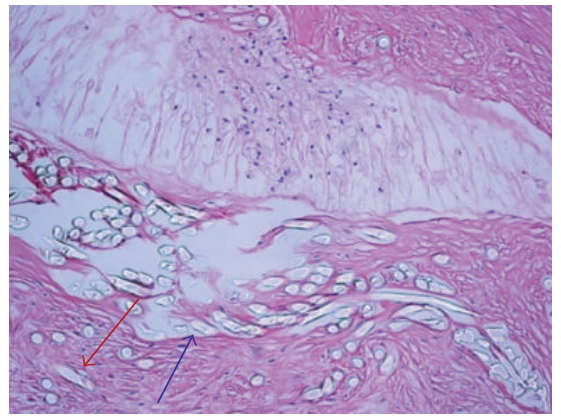

(h)

Figure 5: HE staining of occluder at different time points after procedure (a, c, e, and g original magnification: $10 \times 10$; b, d, f, and h original magnification $10 \times 20)$. (a, b) At 8 weeks after implantation, PDO (black arrow) and PLA (blue arrow) wire was kept in shape; there were a large number of inflammatory cells around the around PDO wire (black arrow). (c, d) At 12 weeks after implantation, the edge of PDO wire (black arrow) began to disintegrate, and newly formed microvessels could be seen (red arrow); PLA wire (blue arrow) was surrounded by inflammatory cells. (e, f) At 16 weeks after implantation, the majority of the edge of PDO has been disintegrated (black arrow); the inflammation has subsided; well remodeling microvessels could be seen (red arrow); aggregates of multinuclear giant cells (yellow arrow) were found in the areas where the PDO wires had undergone degradation. (g, h) At 24 weeks after implantation, PDO almost completes degradation and substituted by hyperplastic fibrous connective tissue (black arrow), the PLA (blue arrow) that was partly degraded and neovascular could be seen (red arrow). 


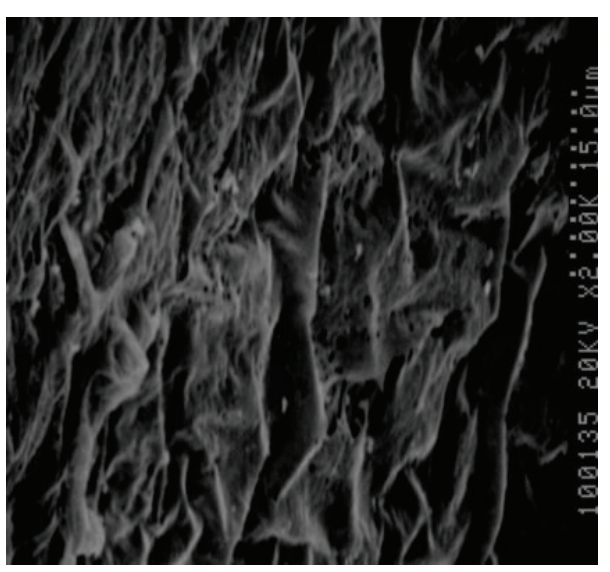

(a)

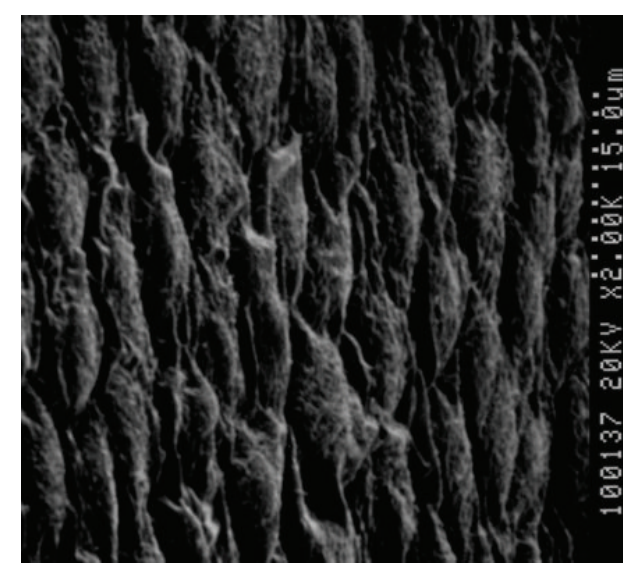

(b)

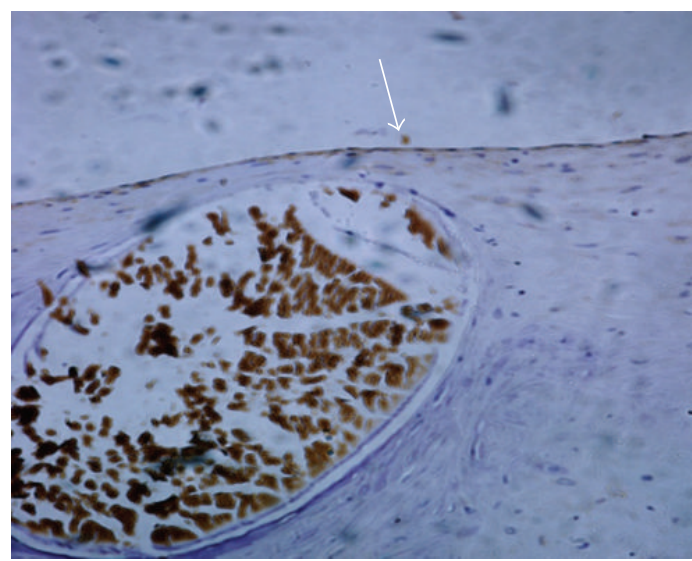

(c)

FIGURE 6: Endothelialization of of the surface of occlude. (a) Eight weeks after the procedure, the surface of occluder was lined with a layer of sparse endothelial cells (scanning electron micrographs $\times 2,000$ ); (b) paving-stone-like arranged endothelial cells cover completely the surfaces of devices at 12 weeks (scanning electron micrographs $\times 2,000$ ); (c) positive immunohistochemical staining (white arrow) for CD34 in superficial cells of the neointima at 12 weeks after implantation (original magnification $10 \times 10$ ).

particles; occluder disc was fully opened according to the commencement of tantalum particles and checked whether the discs were fully opened, thus providing the best solution to the problem of bad biodegradable occluder-DSA image.

In our study, acute ASD model was successfully created in 18 dogs, and 16 occluders were successfully implanted in ASD models, 2 implants failed due to lack of experience. During the closure process, the forceps device of delivery system was firmly bonded with a small circular loop of occluder at the rear, and then occluders were delivered and released smoothly, both of the two discs could change back to discoidal forms after the release, the opening degree was confirmed by ultrasound monitoring and X-ray contrast medium tantalum particles, which demonstrated that biodegradable occluder has a high safety level and simple operability, no obvious complications were found.

PDO has shown excellent biocompatibility in applications such as sutures $[19,20]$, orthopedic tissue fixation devices [21,22], and chest wall prosthesis [23]. In the vasculature, PDO has been tested as vascular grafts [24-26], stents [27], and valved patches [28] and has shown good biocompatibility and minimal thrombosis. The surface endothelialization of occluder has contributed to reduce the risk of thrombosis and endocarditis. Our experiment showed that 8 weeks after procedure, the surface of occluder was covered with reddishwhite film. Immunohistochemical staining of occluder at 12 weeks showed the surface lining of occluder with a continuous layer of endothelial cells (CD34+). Electron microscopic examination of occluder at 12 weeks showed denser endothelial cells and showed a wave-like surface of occluder.

4.1. The Degradation Rate of PDO Was Matching the Speed of Tissue Formation. In the early stages of hydrolytic degradation, water diffuses inside the amorphous regions of PDO wire and causes the formation of tie molecules from the hydrolytic scission of chain segments. The chain segments themselves turned into free flexible molecular segments which were easy to form new orderly folded regions to limit the infiltration of water molecules. Therefore, the destruction of interfibrillar amorphous region was not serious in the initial period; the degradation process of PDO fibers was much gentle, and monofilament fiber displayed adequate tensile strength. They sustained the dormant stage for 90 
days and then entered to the active stage [29, 30]. The PLA film degradation was significantly slower than the PDO, and no significant degradation was found at 12 weeks after procedure. At 24 weeks, PLA film was partially degraded which was conducive to preventing the shedding of tantalum particle and postoperative ASD recanalization. We used PDO wire as the scaffold to provide support and chosen woven PLA as the film to block blood flow and to mediate thrombus formation. Biodegradable occluders were followed up for 6 months after the implantation. Animals were sacrificed at different times; after procedure, the cardiac gross anatomy showed all occluders stable in the interatrial septum, the occluder shape was retained, and there was no PDO wire fracture. Naked-eye examination showed that 12 weeks after the procedure the structural integrity of PDO wire remained visible, at 24 weeks the occluder was been substituted by dense fibrous connective tissue, and there was no postoperative ASD recanalization. At 8 weeks after the procedure, histopathological examination showed that PDO wire retained its shape, at 12 weeks PDO wire at the edge of the discs began to disintegrate, but still in good shape, and at 24 weeks most of the PDO wire had already been disintegrated and substituted by hyperplastic fibrous connective tissue.

In conclusion, from our limited experience, it can be speculated that biodegradable ASD occluders have the advantages of easy surgery, high success rate, fewer complications, and excellent biocompatibility. They are rapid with full endothelialization and will have bright foreground application.

4.2. Study Limitations. The present study has several limitations. It is possible that an acute myocardium injury associated with punch and dilatation could have effect on the healing response after implantation of the occluder. This should be noted when interpreting the results of this study. Occluder size was likely to be limited. The diameter of PDO monofilament fiber was $0.298 \mathrm{~mm}$; nickel-titanium wire was only $0.1 \mathrm{~mm}$; thus, the preparation of bigger and fully biodegradable ASD occluder would certainly cause difficulties. A lot of efforts are required to look after the synthesis of biodegradable materials with thinner diameter and effective support.

Also, being a feasibility study, the dogs were followed up for only 24 weeks. Although the complete endothelialization was achieved in 12 weeks and the PDO framework was largely absorbed at 24 weeks after implantation, we believe that longer followup periods are needed as the PLLA needs 24 months or more to dissolve in vivo.

\section{Authors' Contribution}

Y.-f. Zhu and X.-m. Huang are contributed equally to the paper.

\section{Acknowledgments}

This study was supported by the Shanghai Committee of Science and Technology of China (Grant no. 10411960200) and National Natural Science Foundation of China (Grant no. 81170150).

\section{References}

[1] M. Carminati, S. Giusti, G. Hausdorf et al., "A European multicentric experience using the CardioSEAL $($ and Starflex double umbrella devices to close interatrial communications holes within the oval fossa," Cardiology in the Young, vol. 10, no. 5, pp. 519-526, 2000.

[2] U. Krumsdorf, S. Ostermayer, K. Billinger et al., "Incidence and clinical course of thrombus formation on atrial septal defect and patient foramen ovale closure devices in 1,000 consecutive patients," Journal of the American College of Cardiology, vol. 43, no. 2, pp. 302-309, 2004.

[3] A. Divekar, T. Gaamangwe, N. Shaikh, M. Raabe, and J. Ducas, "Cardiac perforation after device closure of atrial septal defects with the Amplatzer septal occluder," Journal of the American College of Cardiology, vol. 45, no. 8, pp. 1213-1218, 2005.

[4] M. Kitano, S. Yazaki, H. Sugiyama, and O. Yamada, "The influence of morphological changes in amplatzer device on the atrial and aortic walls following transcatheter closure of atrial septal defects," Journal of Interventional Cardiology, vol. 22, no. 1, pp. 83-91, 2009.

[5] E. Ivens, C. Hamilton-Craig, C. Aroney, A. Clarke, H. Jalali, and D. J. Burstow, "Early and late cardiac perforation by amplatzer atrial septal defect and patent foramen ovale devices," Journal of the American Society of Echocardiography, vol. 22, no. 9, pp. 1067-1070, 2009.

[6] D. J. Di Bardino, D. B. McElhinney, A. K. Kaza, and J. E. Mayer Jr, "Analysis of the US food and drug administration manufacturer and user facility device experience database for adverse events involving Amplatzer septal occluder devices and comparison with the society of thoracic surgery congenital cardiac surgery database," Journal of Thoracic and Cardiovascular Surgery, vol. 137, no. 6, pp. 1334-1341, 2009.

[7] D. Duong-Hong, Y. D. Tang, W. Wu et al., "Fully biodegradable septal defect occluder-a double umbrella design," Catheterization and Cardiovascular Interventions, vol. 76, no. 5, pp. 711-718, 2010.

[8] W. Wu, J. Yip, Y. D. Tang et al., "A novel biodegradable septal defect occluder: the, "Chinese Lantern" design, proof of concept," Innovations, vol. 6, no. 4, pp. 221-230, 2011.

[9] C. Jux, H. Bertram, P. Wohlsein, M. Bruegmann, and T. Paul, "Interventional atrial septal defect closure using a totally bioresorbable occluder matrix. Development and preclinical evaluation of the BioSTAR device," Journal of the American College of Cardiology, vol. 48, no. 1, pp. 161-169, 2006.

[10] M. J. Mullen, D. Hildick-Smith, J. V. De Giovanni et al., "BioSTAR evaluation STudy (BEST): a prospective, multicenter, phase I clinical trial to evaluate the feasibility, efficacy, and safety of the BioSTAR bioabsorbable septal repair implant for the closure of atrial-level shunts," Circulation, vol. 114, no. 18, pp. 1962-1967, 2006.

[11] R. Hoehn, C. Hesse, H. Ince, and M. Peuster, "First experience withthe BioSTAR-device for various applications in pediatric patients with congenital heart disease," Catheterization and Cardiovascular Interventions, vol. 75, no. 1, pp. 72-77, 2010.

[12] B. J. Van Den Branden, M. C. Post, H. W. Plokker, J. M. Ten Berg, and M. J. Suttorp, "Patent foramen ovale closure using a bioabsorbable closure device: safety and efficacy at 6-month follow-up," Cardiovascular Interventions, vol. 3, no. 9, pp. 968973, 2010. 
[13] M. Cikirikcioglu, S. Cherian, R. Lerch et al., "Late tamponade secondary to aortic root perforation by biostar septal closure device," Annals of Thoracic Surgery, vol. 91, no. 2, pp. 604-606, 2011.

[14] S. J. Liu, K. M. Peng, C. Y. Hsiao, K. S. Liu, H. T. Chung, and J. K. Chen, "Novel biodegradable polycaprolactone occlusion device combining nanofibrous PLGA/Collagen membrane for closure of atrial septal defect (ASD)," Annals of Biomedical Engineering, pp. 1-8, 2011.

[15] M. Zilberman, K. D. Nelson, and R. C. Eberhart, "Mechanical properties and in vitro degradation of bioresorbable fibers and expandable fiber-based stents," Journal of Biomedical Materials Research B, vol. 74, no. 2, pp. 792-799, 2005.

[16] I. Battersby and R. Doyle, "Use of a biodegradable self-expanding stent in the management of a benign oesophageal stricture in a cat," Journal of Small Animal Practice, vol. 51, no. 1, pp. 49-52, 2010.

[17] S. M. Stivaros, L. R. Williams, C. Senger, L. Wilbraham, and H. U. Laasch, "Woven polydioxanone biodegradable stents: a new treatment option for benign and malignant oesophageal strictures," European Radiology, vol. 20, no. 5, pp. 1069-1072, 2010.

[18] R. Lischke, J. Pozniak, D. Vondrys, and M. J. Elliott, "Novel biodegradable stents in the treatment of bronchial stenosis after lung transplantation," European Journal of Cardio-thoracic Surgery, 2011.

[19] J. N. Im, J. K. Kim, H. K. Kim, C. H. In, K. Y. Lee, and W. H. Park, "In vitro and in vivo degradation behaviors of synthetic absorbable bicomponent monofilament suture prepared with poly(p-dioxanone) and its copolymer," Polymer Degradation and Stability, vol. 92, no. 4, pp. 667-674, 2007.

[20] M. Kosan, U. Gonulalan, B. Ozturk et al., "Tissue reactions of suture materials (polyglactine 910, chromed catgut and polydioxanone) on rat bladder wall and their role in bladder stone formation," Urological Research, vol. 36, no. 1, pp. 43-49, 2008.

[21] R. B. Cady, J. A. Siegel, G. Mathien, J. A. Spadaro, and S. E. Chase, "Physeal response to absorbable polydioxanone bone pins in growing rabbits," Journal of Biomedical Materials Research, vol. 48, no. 3, pp. 211-215, 1999.

[22] H. Pihlajamäki, S. Salminen, O. Laitinen, O. Tynninen, and O. Böstman, "Tissue response to polyglycolide, polydioxanone, polylevolactide, and metallic pins in cancellous bone: an experimental study on rabbits," Journal of Orthopaedic Research, vol. 24, no. 8, pp. 1597-1606, 2006.

[23] X. Qin, H. Tang, Z. Xu et al., "Chest wall reconstruction with two types of biodegradable polymer prostheses in dogs," European Journal of Cardio-thoracic Surgery, vol. 34, no. 4, pp. 870 $874,2008$.

[24] S. A. Sell, M. J. McClure, C. P. Barnes et al., "Electrospun polydioxanoneelastin blends: potential for bioresorbable vascular grafts," Biomedical Materials, vol. 1, no. 2, pp. 72-80, 2006.

[25] V. Thomas, X. Zhang, and Y. K. Vohra, "A biomimetic tubular scaffold with spatially designed nanofibers of Protein/PDS $B$ bio-blends," Biotechnology and Bioengineering, vol. 104, no. 5, pp. 1025-1033, 2009.

[26] P. S. Wolfe, P. Madurantakam, K. Garg, S. A. Sell, M. J. Beckman, and G. L. Bowlin, "Evaluation of thrombogenic potential of electrospun bioresorbable vascular graft materials: acute monocyte tissue factor expression," Journal of Biomedical Materials Research A, vol. 92, no. 4, pp. 1321-1328, 2010.

[27] P. Zamiri, Y. Kuang, U. Sharma et al., "The biocompatibility of rapidly degrading polymeric stents in porcine carotid arteries," Biomaterials, vol. 31, no. 31, pp. 7847-7855, 2010.

[28] D. Kalfa, A. Bel, A. Chen-Tournoux et al., "A polydioxanone electrospun valved patch to replace the right ventricular outflow tract in a growing lamb model," Biomaterials, vol. 31, no. 14, pp. 4056-4063, 2010.

[29] M. A. Sabino, S. González, L. Márquez, and J. L. Feijoo, "Study of the hydrolytic degradation of polydioxanone PPDX," Polymer Degradation and Stability, vol. 69, no. 2, pp. 209-216, 2000.

[30] M. A. Sabino, J. Albuerne, A. Müller, J. Brisson, and R. E. Prud'homme, "Influence of in vitro hydrolytic degradation on the morphology and crystallization behavior of poly(p-dioxanone)," Biomacromolecules, vol. 5, no. 2, pp. 358-370, 2004. 

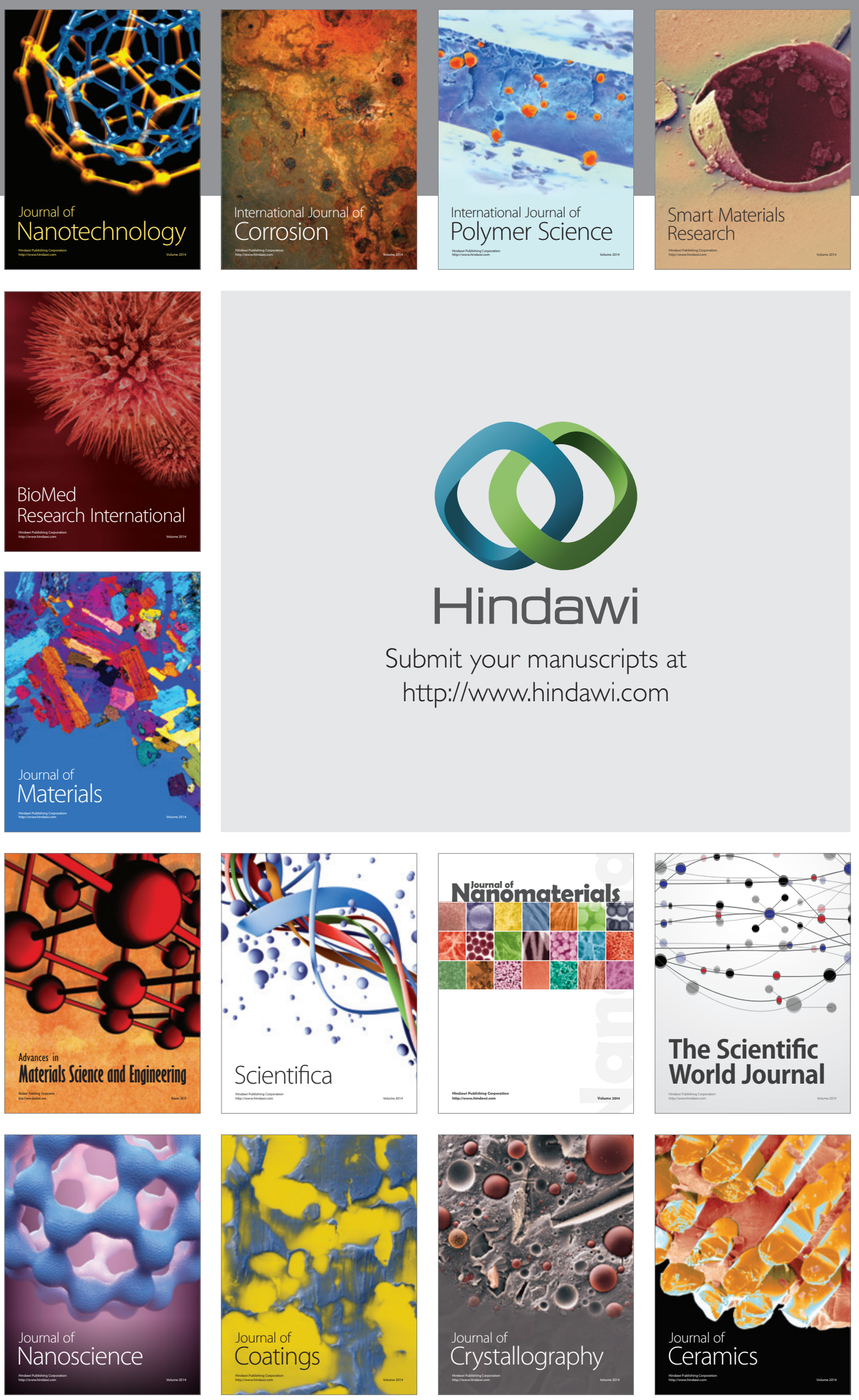

The Scientific World Journal

Submit your manuscripts at

http://www.hindawi.com

\section{World Journal}

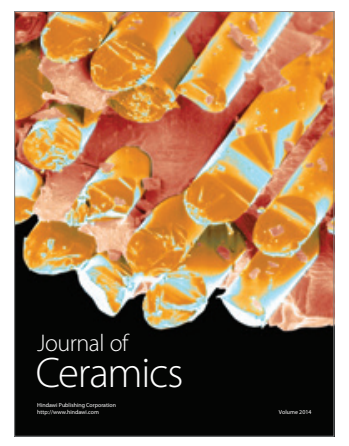

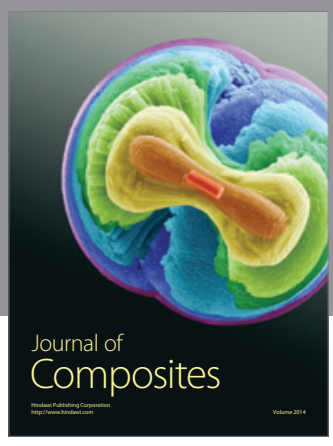
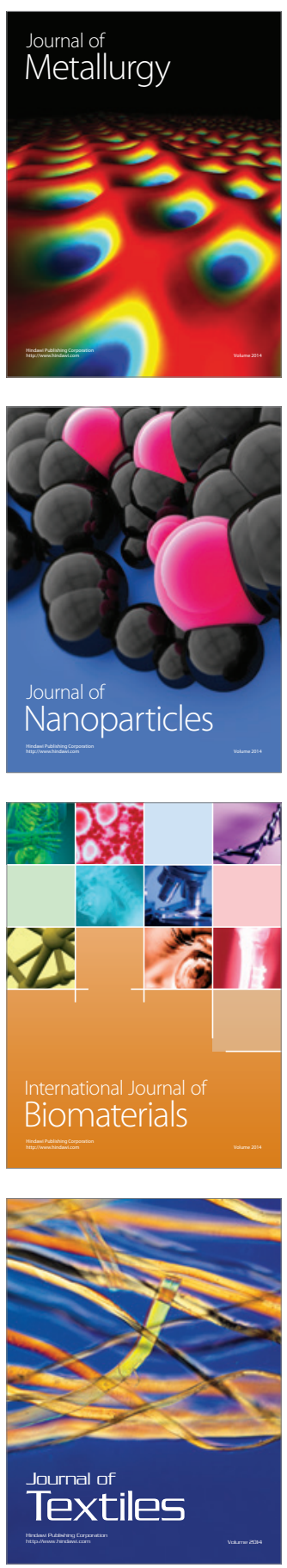